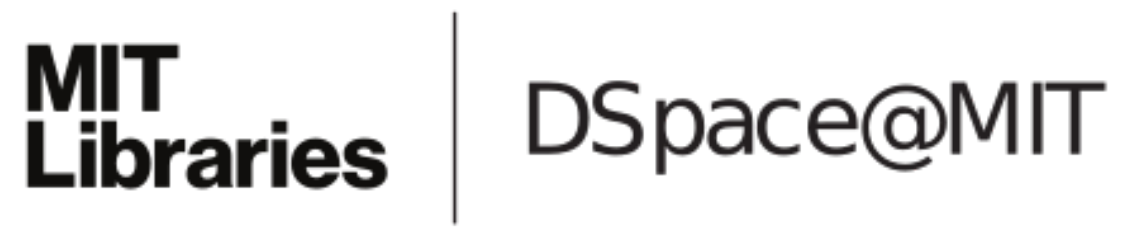

\author{
MIT Open Access Articles
}

Error-corrected quantum sensing

The MIT Faculty has made this article openly available. Please share how this access benefits you. Your story matters.

Citation: Zhou, Sisi et al. "Error-corrected quantum sensing." Proceedings Volume 10934, Optical, Opto-Atomic, and Entanglement-Enhanced Precision Metrology, February 2019, San Francisco, USA, edited by Selim M. Shahriar and Jacob Scheuer for SPIE, 2019. (C) 2019 SPIE

\section{As Published: $10.1117 / 12.2511587$}

Publisher: Society of Photo-Optical Instrumentation Engineers (SPIE)

Persistent URL: https://hdl.handle.net/1721.1/124357

Version: Final published version: final published article, as it appeared in a journal, conference proceedings, or other formally published context

Terms of Use: Article is made available in accordance with the publisher's policy and may be subject to US copyright law. Please refer to the publisher's site for terms of use. 


\section{Error-corrected quantum sensing}

Zhou, Sisi, Layden, David, Zhang, Mengzhen, Preskill, John, Cappellaro, Paola, et al.

Sisi Zhou, David Layden, Mengzhen Zhang, John Preskill, Paola Cappellaro, Liang Jiang, "Error-corrected quantum sensing," Proc. SPIE 10934, Optical, Opto-Atomic, and Entanglement-Enhanced Precision Metrology, 109341J (1 March 2019); doi: 10.1117/12.2511587

SPIE. Event: SPIE OPTO, 2019, San Francisco, California, United States 


\title{
Error-corrected quantum sensing
}

\author{
Sisi Zhou ${ }^{1,2}$, David Layden ${ }^{3}$, Mengzhen Zhang ${ }^{1,2}$, John Preskill ${ }^{4}$, Paola Cappellaro ${ }^{3}$, and Liang \\ Jiang ${ }^{1,2}$ \\ ${ }^{1}$ Yale Quantum Institute, Yale University, New Haven, CT 06511, USA \\ ${ }^{2}$ Departments of Applied Physics and Physics, Yale University, New Haven, CT 06511, USA \\ ${ }^{3}$ Research Laboratory of Electronics and Department of Nuclear Science and Engineering, \\ Massachusetts Institute of Technology, Cambridge, MA 02139, USA \\ ${ }^{4}$ Institute for Quantum Information and Matter, California Institute of Technology, Pasadena, \\ CA 91125, USA
}

\begin{abstract}
Quantum metrology has many important applications in science and technology, ranging from frequency spectroscopy to gravitational wave detection. Quantum mechanics imposes a fundamental limit on measurement precision, called the Heisenberg limit, which can be achieved for noiseless quantum systems, but is not achievable in general for systems subject to noise. Here we study how measurement precision can be enhanced through quantum error correction, a general method for protecting a quantum system from the damaging effects of noise. We find a necessary and sufficient condition for achieving the Heisenberg limit using quantum probes subject to Markovian noise, assuming that noiseless ancilla systems are available, and that fast, accurate quantum processing can be performed. When the sufficient condition is satisfied, the quantum error-correcting code achieving the best possible precision can be found by solving a semidefinite program. We also show that noiseless ancilla are not needed when the signal Hamiltonian and the error operators commute. Finally we provide two explicit, archetypal examples of quantum sensors: qubits undergoing dephasing and a lossy bosonic mode.
\end{abstract}

\section{INTRODUCTION}

Quantum metrology concerns the task of estimating a parameter, or several parameters, characterizing the Hamiltonian of a quantum system ${ }^{1-3}$. It is of great importance in science and technology, with wide applications including frequency spectroscopy, magnetometry, accelerometry, gravimetry, gravitational wave detection, and other high-precision measurements ${ }^{4-13}$. However, as with most quantum technologies, the performance of quantum sensors is limited by decoherence. Typically, a quantum sensor acquires a signal as a relative phase between two states in coherent superposition. Its sensitivity therefore depends both on how quickly this phase accumulates, and on how long the superposition remains coherent. The fundamental strategy to enhance sensitivity is then to increase the rate of signal acquisition (e.g., by exploiting entanglement) without reducing the coherence time by an equal amount ${ }^{14}$. These competing demands pose a familiar dilemma in quantum engineering: a quantum sensor must couple strongly to its environment without being rapidly decohered by it.

The Heisenberg limit (HL) constrains how the precision of parameter estimation improves as the total probing time $t$ increases. According to HL, the scaling of precision with $t$ can be no better than $1 / t$; equivalently, precision scales no better than $1 / N$ with the total number of probes $N$ used in an experiment. For a noiseless system, HL scaling is attainable in principle by preparing an entangled "cat" state of $N$ probes ${ }^{12}$. Due to decoherence, precision usually scales like $1 / \sqrt{N}$ in practice, called the standard quantum limit (SQL), which can be achieved by using $N$ independent probes ${ }^{14-19}$. The quest for measurement schemes surpassing the SQL has inspired a variety of clever strategies, such as squeezing the vacuum ${ }^{4}$, optimizing the probing time ${ }^{20}$, monitoring the environment ${ }^{21-23}$, and exploiting non-Markovian effects ${ }^{24-26}$.

Quantum error correction (QEC) is a method for reducing noise in quantum channels and quantum processors $^{27-29}$. In principle, it enables a noisy quantum computer to simulate faithfully an ideal quantum computer, with reasonable overhead cost, if the noise is not too strong or too strongly correlated. Recently, quantum error correction (QEC) has emerged as a particularly powerful tool for enhancing the precision in quantum metrology $^{30-41}$. When a signal and a noise source are coupled to a sensor in orthogonal directions, e.g., through $\sigma_{z}$ and

Optical, Opto-Atomic, and Entanglement-Enhanced Precision Metrology, edited by Selim M. Shahriar,

Jacob Scheuer, Proc. of SPIE Vol. 10934, 109341J · C 2019 SPIE · CCC

code: $0277-786 \mathrm{X} / 19 / \$ 18 \cdot$ doi: $10.1117 / 12.2511587$

Proc. of SPIE Vol. 10934 109341J-1 
$\sigma_{x}$, it was shown that HL scaling could be restored asymptotically (in the sense that recoveries are performed with sufficiently high frequency) via a two-qubit code utilizing one probing qubit and one noiseless ancillary qubit $^{30-33}$.

QEC has found its application in several important sensing scenarios, but its potential value in quantum metrology has not yet been fully fleshed out, even as a matter of principle. As is the case for quantum computing, we should expect positive (or negative) statements about improving metrology via QEC to be premised on suitable assumptions about the properties of the noise and the capabilities of our quantum hardware. A serious obstacle for applications of QEC to sensing is that it may in some cases be exceedingly hard to distinguish the signal arising from the Hamiltonian evolution of the probe system from the effects of the noise acting on the probe.

In this paper, we summarize our results in Refs. 38,41, revealing how and when QEC codes could restore HL scaling in quantum sensing. Let us first state our assumptions about the physical system. We assume that the probes used for parameter estimation are subject to noise described by a Markovian master equation ${ }^{42,43}$, where the strength and structure of this noise is beyond the experimentalist's control. Aside from the probe system, the experimentalist also has noiseless ancilla qubits at her disposal, and the ability to apply noiseless quantum gates which act jointly on the ancilla and probe; she can also perform perfect ancilla measurements, and reset the ancillas after measurement. Later on, we will also see that noiseless ancillas are not needed when the signal Hamiltonian and the error operators commute: a practically relevant type of decoherence in quantum sensors. Furthermore, we assume that a quantum gate or measurement can be executed in an arbitrarily short time (though the Markovian description of the probe's noise is assumed to be applicable no matter how fast the processing).

We endow the experimentalist with these powerful tools because we wish to address, as a matter of principle, how effectively QEC can overcome the deficiencies of the noisy probe system. Our scenario may be of practical interest as well, in hybrid quantum systems where ancillas are available which have a much longer coherence time than the probe. For example, sensing of a magnetic field with a probe electron spin can be enhanced by using a quantum code which takes advantage of the long coherence time of a nearby (ancilla) nuclear spin in diamond ${ }^{44}$. In cases where noise acting on the ancilla is weak but not completely negligible, we could use QEC to enhance the coherence time of the ancilla, thus providing a justification for our idealized setting in which the ancilla is effectively noiseless ${ }^{38}$. Recent experimental progress also indicates that fast quantum error correction is applicable in at least some realistic settings. For example, in superconducting devices QEC has reached the break-even point where the lifetime of an encoded qubit exceeds the natural lifetime of the constituents of the system $^{45}$; one- and two-qubit logical operations have also been demonstrated ${ }^{46,47}$. Moreover, if sensing could be performed using a probe encoded within a noiseless subspace or subsystem ${ }^{48}$ or protected by autonomous quantum error correction ${ }^{49,50}$, then active error correction would not be needed to protect the probe, making the QEC scheme more feasible using near-term technology.

In accord with our assumptions, in Sec. 2 we first state a necessary and sufficient condition for achieving HL scaling in a finite-dimensional system, which we call the HNLS condition, or simply HNLS, an acronym for "Hamiltonian not in Lindblad span". The condition is formulated as an algebraic relation between the signal Hamiltonian whose coefficient is to be estimated and the Lindblad operators $\left\{L_{k}\right\}$ which appear in the master equation describing the evolution of the probe. For the case where our sufficient condition is satisfied, we explicitly construct a QEC code which achieves HL scaling.

We also show that the problem of finding the QEC code achieving the best possible precision can be formulated as a semidefinite program that can be efficiently solved numerically in Sec. 3. Furthermore, when the signal Hamiltonian and the error operators commute, the semidefinite program can be modified to find the optimal QEC code which does not use noiseless ancilla. In Sec. 4, we present the optimal code in two explicit, archetypal examples of quantum sensors: qubits undergoing dephasing and a lossy bosonic mode.

In Sec. 5, we consider the case when HNLS is not satisfied, for example when the noise channel is full rank, the HL scaling cannot be achieved. However, for noise which is $\epsilon$-close to meeting our criterion, using the QEC code ensures that HL scaling can be maintained approximately for a time $O(1 / \epsilon)$, before crossing over to asymptotic SQL scaling. In addition, we calculate the precision limit beyond HNLS for qubits under correlated dephasing noise. 
(a)

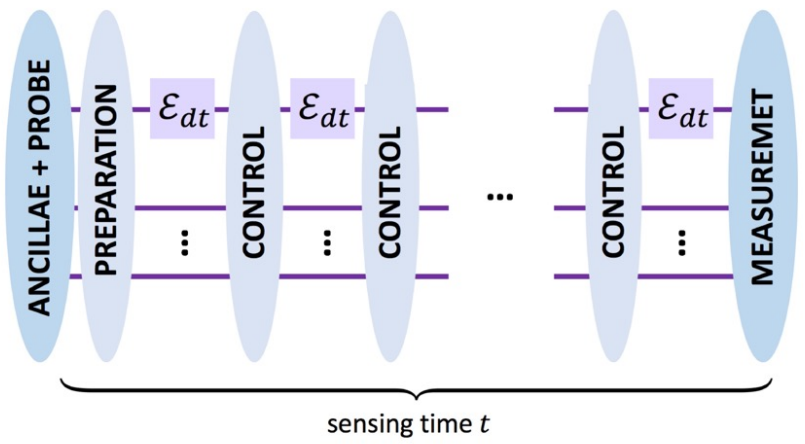

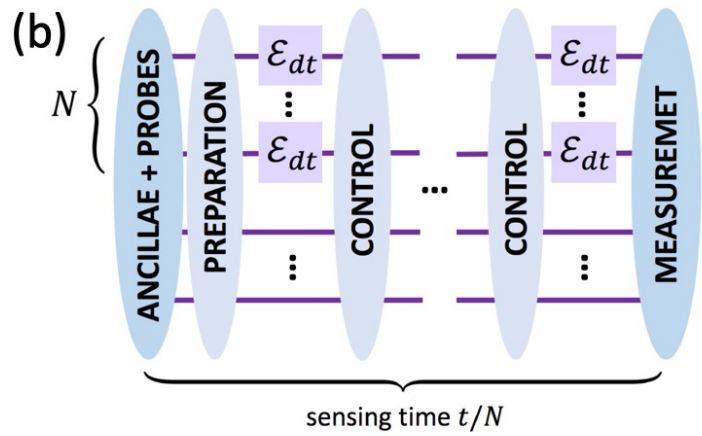

Figure 1. Metrology schemes. (a) The sequential scheme. One probe sequentially senses the parameter for time $t$, with quantum controls applied every $d t$. (b) The parallel scheme. $N$ probes sense the parameter for time $t / N$ in parallel. The parallel scheme can be simulated by the sequential scheme.

\section{THE HNLS CONDITION}

To derive the HNLS condition, we adopt the sequential scheme for quantum metrology ${ }^{36,51,52}$ (see Fig. 1a). In this scheme, a single noisy probe senses the unknown parameter for many rounds, where each round lasts for a short time interval $d t$, and the total number of rounds is $t / d t$, where $t$ is the total sensing time. In between rounds, an arbitrary (noiseless) quantum operation can be applied instantaneously, which acts jointly on the probe and the noiseless ancillas. The rapid operations between rounds empower us to perform QEC, suppressing the damaging effects of the noise on the probe. The sequential scheme is one of the most general metrology scheme, as it can simulate a parallel scheme (Fig. 1b), in which $N$ probes simultaneously sense the parameter for time $t / N^{36,51}$.

We denote the $d$-dimensional Hilbert space of our probe by $\mathcal{H}_{P}$, and we assume the state $\rho_{P}$ of the probe evolves according to a time-homogeneous Lindblad master equation of the form (with $\hbar=1$ ), ${ }^{42,43}$

$$
\frac{d \rho_{P}}{d t}=-i\left[\omega H, \rho_{P}\right]+\sum_{k=1}^{r}\left(L_{k} \rho_{P} L_{k}^{\dagger}-\frac{1}{2}\left\{L_{k}^{\dagger} L_{k}, \rho_{P}\right\}\right)
$$

where $\omega H$ is the Hamiltonian from which $\omega$ is to be estimated, $\left\{L_{k}\right\}$ are the Lindblad jump operators, and $r$ is the "rank" of the noise channel acting on the probe (the smallest number of Lindblad operators needed to describe the channel). We denote by $\mathcal{H}_{A}$ another $d$-dimensional Hilbert space of a noiseless ancilla system. Over the small time interval $d t$, during which no controls are applied, the ancilla evolves trivially, and the joint state $\rho$ of probe and ancilla evolves according to the quantum channel

$$
\mathcal{E}_{d t}(\rho)=\rho-i[\omega H, \rho] d t+\sum_{k=1}^{r}\left(L_{k} \rho L_{k}^{\dagger}-\frac{1}{2}\left\{L_{k}^{\dagger} L_{k}, \rho\right\}\right) d t+O\left(d t^{2}\right)
$$

where $\rho \in \mathcal{H}_{P} \otimes H_{A}$ and $H, L_{k}$ are shorthand for $H \otimes I, L_{k} \otimes I$ respectively. We assume that this time interval $d t$ is sufficiently small that corrections higher order in $d t$ can be neglected. In between rounds of sensing, each lasting for time $d t$, control operations acting on $\rho$ are applied instantaneously.

We denote by $\mathfrak{S}$ the real linear subspace of Hermitian operators spanned by the operators $I, L_{k}, L_{k}^{\dagger}, L_{k}^{\dagger} L_{j}$ (for all $k$ and $j$ ranging from 1 to $r$ ), and say that the Hamiltonian $H$ obeys the HNLS condition if $H$ is not contained in the Lindblad span $\mathfrak{S}$. Now we state our main conclusion about parameter estimation using fast and accurate quantum controls as Theorem $1^{37,38}$ and sketch the proof in the following two subsections.

Theorem 1 (HNLS) Consider a finite-dimensional probe with Hamiltonian $\omega H$, subject to Markovian noise described by a Lindblad master equation with jump operators $\left\{L_{k}\right\}_{k=1}^{r}$. Then $\omega$ can be estimated with HL (Heisenberg-limited) precision if and only if $H$ and $\left\{L_{k}\right\}$ obey the HNLS condition. 


\subsection{Non-achievability of HL when HNLS fails}

The necessary condition for HL scaling can be derived from the quantum Cramér-Rao bound ${ }^{53-55}$

$$
\delta \hat{\omega} \geq \frac{1}{\sqrt{N_{\operatorname{expr}} \cdot F\left(\rho_{\omega}(t)\right)}} .
$$

Here $\hat{\omega}$ denotes any unbiased estimator for the parameter $\omega$, and $\delta \hat{\omega}$ is that estimator's standard deviation, or precision of parameter estimation. $F\left(\rho_{\omega}(t)\right)$ is the quantum Fisher information (QFI) of the state $\rho_{\omega}(t)$; this state is obtained by preparing an initial state $\rho_{\text {in }}$ of the probe, and then evolving this state for total time $t$, where the evolution is governed by Eq. (1) and our fast quantum controls. For a scheme in which the measurement protocol is repeated many times in succession, $N_{\text {expr }}$ denotes the number of such repetitions.

We show that $F\left(\rho_{\omega}(t)\right)$ is at most asymptotically linear in $t$ when the Hamiltonian $H \in \mathfrak{S}$, which means that SQL scaling cannot be surpassed in this case. Though it is challenging to compute the maximum attainable QFI for arbitrary quantum channels, useful upper bounds on QFI can be derived, which provide lower bounds on the precision of quantum metrology ${ }^{16-19,36,51,56}$. The quantum channel describing the joint evolution of probe and ancilla has a Kraus operator representation $\mathcal{E}_{d t}(\rho)=\sum_{k} K_{k} \rho K_{k}^{\dagger}$, and in terms of these Kraus operators we define

$$
\alpha_{d t}=\sum_{k} \dot{K}_{k}^{\dagger} \dot{K}_{k}=\dot{\mathbf{K}}^{\dagger} \dot{\mathbf{K}}, \quad \beta_{d t}=i \sum_{k} \dot{K}_{k}^{\dagger} K_{k}=i \dot{\mathbf{K}}^{\dagger} \mathbf{K}
$$

where we express the Kraus operators in vector notation $\mathbf{K}:=\left(K_{0}, K_{1}, \cdots\right)^{T}$, and the over-dot means the derivative with respect to $\omega$. If $\rho_{\text {in }}$ is the initial joint state of probe and ancilla at time 0 , and $\rho_{\omega}(t)$ is the corresponding state at time $t$, then the upper bound on the QFI

$$
F\left(\rho_{\omega}(t)\right) \leq 4 \frac{t}{d t}\left\|\alpha_{d t}\right\|+4\left(\frac{t}{d t}\right)^{2}\left\|\beta_{d t}\right\|\left(\left(\left\|\beta_{d t}\right\|+2 \sqrt{\left\|\alpha_{d t}\right\|}\right)\right.
$$

$\left(\|\cdot\|\right.$ denotes the operator norm) derived by the "channel extension method" holds for any choice of $\rho_{\text {in }}$ even when fast and accurate quantum controls are applied during the evolution ${ }^{36}$. This upper bound on the QFI provides a lower bound on the precision $\delta \hat{\omega}$ via Eq. (3).

Kraus representations are not unique - for any matrix $u$ satisfying $u^{\dagger} u=I, \mathbf{K}^{\prime}=u \mathbf{K}$ represents the same channel as K. Hence, we can tighten the upper bound on the QFI by minimizing the RHS of Eq. (5) over all such valid Kraus representations. We see that $\dot{\mathbf{K}}^{\prime}=u(\dot{\mathbf{K}}-i h \mathbf{K}), \dot{\mathbf{K}}^{\dagger}=(\dot{\mathbf{K}}-i h \mathbf{K})^{\dagger} u^{\dagger}$ where $h=i u^{\dagger} \dot{u}$. Therefore, to find $\alpha_{d t}$ and $\beta_{d t}$ providing the tightest upper bound on the QFI, it suffices to replace $\dot{\mathbf{K}}$ by $\dot{\mathbf{K}}-i h \mathbf{K}$ and to optimize over the Hermitian matrix $h$. To evaluate the bound for asymptotically large $t$, we expand $\alpha_{d t}, \beta_{d t}, h$ in powers of $\sqrt{d t}$ :

$$
\begin{aligned}
\alpha_{d t} & =\alpha^{(0)}+\alpha^{(1)} \sqrt{d t}+\alpha^{(2)} d t+O\left(d t^{3 / 2}\right), \\
\beta_{d t} & =\beta^{(0)}+\beta^{(1)} \sqrt{d t}+\beta^{(2)} d t+\beta^{(3)} d t^{3 / 2}+O\left(d t^{2}\right), \\
h & =h^{(0)}+h^{(1)} \sqrt{d t}+h^{(2)} d t+h^{(3)} d t^{3 / 2}+O\left(d t^{2}\right) .
\end{aligned}
$$

It can be shown that the first two terms in $\alpha_{d t}$ and the first four terms in $\beta_{d t}$ can all be set to zero by choosing a suitable $h$, assuming that HNLS is violated ${ }^{37,38}$. We therefore have $\alpha_{d t}=O(d t)$ and $\beta_{d t}=O\left(d t^{2}\right)$, so that the second term in the RHS of Eq. (5) vanishes as $d t \rightarrow 0$ :

$$
F\left(\rho_{\omega}(t)\right) \leq 4\left\|\alpha^{(2)}\right\| t
$$

proving that SQL scaling cannot be surpassed when HNLS is violated (the necessary condition in Theorem 1). We require the probe to be finite dimensional in the statement of Theorem 1 because otherwise the norm of $\alpha_{d t}$ or $\beta_{d t}$ could be infinite. 


\subsection{QEC code for HL scaling when HNLS holds}

To prove the sufficient condition for HL scaling, we show that a QEC code achieving HL scaling can be explicitly constructed if $H \notin \mathfrak{S}$. We first consider the QEC condition such that a unitary channel could be recovered. Let $\Pi_{C}$ denote the projection onto the code space,

$$
\begin{aligned}
& \text { [1] } \Pi_{C} L_{k} \Pi_{C}=\lambda_{k} \Pi_{C}, \forall k, \\
& {[2] \Pi_{C} L_{k}^{\dagger} L_{j} \Pi_{C}=\mu_{k j} \Pi_{C}, \forall k, j,}
\end{aligned}
$$

must be satisfied for some complex numbers $\lambda_{k}$ and $\mu_{k j}{ }^{38,57}$. We say the code corrects the Lindblad span $\mathfrak{S}$ if Eq. (10) and Eq. (11) are satisfied. Then the error-corrected joint state of probe and ancilla evolves according to the unitary channel (asymptotically) $)^{38}$

$$
\frac{d \rho}{d t}=-i\left[\omega H_{\mathrm{eff}}, \rho\right]
$$

where $H_{\text {eff }}=\Pi_{C} H \Pi_{C}$. There is a code state for which the evolution depends nontrivially on $\omega$ provided that

$$
\text { [3] } \Pi_{C} H \Pi_{C} \neq \text { constant } \times \Pi_{C} .
$$

For this noiseless evolution with effective Hamiltonian $\omega H_{\text {eff }}$, the QFI of the encoded state at time $t$ is

$$
F\left(\rho_{\omega}(t)\right)=4 t^{2}\left[\operatorname{Tr}\left(\rho_{\text {in }} H_{\text {eff }}^{2}\right)-\left(\operatorname{Tr}\left(\rho_{\text {in }} H_{\text {eff }}\right)\right)^{2}\right],
$$

where $\rho_{\text {in }}$ is the initial state at time $t=0$. The QFI is maximized by choosing the initial pure state

$$
\left|\psi_{\text {in }}\right\rangle=\frac{1}{\sqrt{2}}\left(\left|\lambda_{\min }\right\rangle+\left|\lambda_{\max }\right\rangle\right),
$$

where $\left|\lambda_{\min }\right\rangle,\left|\lambda_{\max }\right\rangle$ are the eigenstates of $H_{\text {eff }}$ with the minimal and maximal eigenvalues; with this choice the QFI is

$$
F\left(\rho_{\omega}(t)\right)=t^{2}\left(\lambda_{\max }-\lambda_{\min }\right)^{2} .
$$

By measuring in the appropriate basis at time $t$, we can estimate $\omega$ with a precision that saturates the CramérRao bound in the asymptotic limit of a large number of measurements, hence realizing HL scaling.

To prove the sufficient condition in Theorem 1, we will now show that a code with properties [1]-[3] can be constructed whenever HNLS is satisfied. To see how the code is constructed, note that the $d$-dimensional Hermitian matrices form a real Hilbert space where the inner product of two matrices $A$ and $B$ is defined to be $\operatorname{Tr}(A B)$. Then $H$ has a unique decomposition into $H=H_{\|}+H_{\perp}$, where $H_{\|} \in \mathfrak{S}$ and $H_{\perp} \perp \mathfrak{S}^{\perp}$, the orthogonal complement of $\mathfrak{S}$. If HNLS holds, then $H_{\perp}$ is nonzero. It must also be traceless, in order to be orthogonal to $I$, which is contained in $\mathfrak{S}$. Therefore, using the spectral decomposition, we can write $H_{\perp}=\frac{1}{2}\left(\operatorname{Tr}\left|H_{\perp}\right|\right)\left(\rho_{0}-\rho_{1}\right)$, where $\rho_{0}$ and $\rho_{1}$ are trace-one positive matrices with orthogonal support and $\left|H_{\perp}\right|:=\sqrt{H_{\perp}^{2}}$.

We can choose our QEC code to be the two-dimensional subspace of $\mathcal{H}_{P} \otimes \mathcal{H}_{A}$ spanned by $\left|0_{\mathrm{L}}\right\rangle$ and $\left|1_{\mathrm{L}}\right\rangle$, which are normalized purifications of $\rho_{0}$ and $\rho_{1}$ respectively, with orthogonal support in $\mathcal{H}_{A}$. (If the probe is $d$-dimensional, a $d$-dimensional ancilla can purify its state.) Properties [1]-[3] could be verified directly. In particular, we have

$$
\left\langle 0_{\mathrm{L}}|H| 0_{\mathrm{L}}\right\rangle-\left\langle 1_{\mathrm{L}}|H| 1_{\mathrm{L}}\right\rangle=\frac{2 \operatorname{Tr}\left(H_{\perp}^{2}\right)}{\operatorname{Tr}\left|H_{\perp}\right|} .
$$

\section{CODE OPTIMIZATION}

When HNLS is satisfied, we can use our QEC code, along with fast and accurate quantum control, to achieve noiseless evolution of the error-corrected probe, governed by the effective Hamiltonian. Because the optimal 
initial state Eq. (15) is a superposition of just two eigenstates of $H_{\text {eff }}$, a two-dimensional QEC code suffices for achieving the best possible precision. For a code with basis states $\left\{\left|0_{\mathrm{L}}\right\rangle,\left|1_{\mathrm{L}}\right\rangle\right\}$, the effective Hamiltonian is

$$
H_{\text {eff }}=\left|0_{\mathrm{L}}\right\rangle\left\langle 0_{\mathrm{L}}\left|H_{\perp}\right| 0_{\mathrm{L}}\right\rangle\left\langle 0_{\mathrm{L}}|+| 1_{\mathrm{L}}\right\rangle\left\langle 1_{\mathrm{L}}\left|H_{\perp}\right| 1_{\mathrm{L}}\right\rangle\left\langle 1_{\mathrm{L}}\right| \text {. }
$$

To search for a better code, with basis states $\left\{\left|0_{\mathrm{L}}\right\rangle,\left|1_{\mathrm{L}}\right\rangle\right\}$, define

$$
\tilde{\rho}_{0}=\operatorname{Tr}_{A}\left(\left|0_{\mathrm{L}}\right\rangle\left\langle 0_{\mathrm{L}}\right|\right), \quad \tilde{\rho}_{1}=\operatorname{Tr}_{A}\left(\left|1_{\mathrm{L}}\right\rangle\left\langle 1_{\mathrm{L}}\right|\right),
$$

and consider

$$
\tilde{H}=\tilde{\rho}_{0}-\tilde{\rho}_{1}
$$

Properties [1]-[2] on the code imply

$$
\operatorname{Tr}(\tilde{H} O)=0, \forall O \in \mathfrak{S}
$$

and we want to maximize

$$
\lambda_{\max }-\lambda_{\min }=\operatorname{Tr}\left(H_{\mathrm{eff}}\left(\left|0_{\mathrm{L}}\right\rangle\left\langle 0_{\mathrm{L}}|-| 1_{\mathrm{L}}\right\rangle\left\langle 1_{\mathrm{L}}\right|\right)\right)=\operatorname{Tr}\left(H_{\perp} \tilde{H}\right),
$$

over matrices $\tilde{H}$ of the form Eq. (20) subject to Eq. (21). $\tilde{H}$ is the difference of two normalized density operators, and satisfies $\operatorname{Tr}(|\tilde{H}|) \leq 2$. In fact, though, if $\tilde{H}$ obeys the constraint Eq. (21), the constraint is still satisfied if we rescale $\tilde{H}$ by a real constant greater than one, which increases $\operatorname{Tr}\left(H_{\perp} \tilde{H}\right)$; hence the maximum of $\operatorname{Tr}\left(H_{\perp} \tilde{H}\right)$ is achieved for $\operatorname{Tr}(|\tilde{H}|)=2$, which means that the optimal $\tilde{\rho}_{0}$ and $\tilde{\rho}_{1}$ should have orthogonal support.

\subsection{Code optimization as a semidefinite program}

Optimization of the QEC code can be formulated as the following optimization problem:

$$
\begin{aligned}
& \text { maximize } \operatorname{Tr}\left(\tilde{H} H_{\perp}\right) \\
& \text { subject to } \operatorname{Tr}(|\tilde{H}|) \leq 2 \text { and } \operatorname{Tr}(\tilde{H} S)=0, \forall S \in \mathfrak{S} .
\end{aligned}
$$

This optimization problem is convex (because $\operatorname{Tr}(|\cdot|)$ is convex) and can be solved by solving its Lagrange dual problem $^{58}$. The optimal QFI has a geometrical interpretation. We find that, for the optimal QEC code, the QFI is $^{38,58}$

$$
F_{\text {opt }}\left(\rho_{\omega}(t)\right)=4 t^{2} \min _{\tilde{H}_{\|} \in \mathfrak{S}}\left\|H-\tilde{H}_{\|}\right\|^{2} \equiv 4 t^{2}\|H-\mathfrak{S}\|^{2},
$$

In this sense, the QFI is determined by the minimal distance between $H$ and $\mathfrak{S}$. The optimal code could be found by the following procedure. We denote by $\tilde{H}_{\|}^{\diamond}$ the choice of $\tilde{H}_{\|} \in \mathfrak{S}$ that minimizes Eq. (24), and we define

$$
\tilde{H}^{\diamond}:=H-\tilde{H}_{\|}^{\diamond}
$$

Then $\tilde{H}^{*}$ which maximizes Eq. (22) has the form

$$
\tilde{H}^{*}=\tilde{\rho}_{0}^{\diamond}-\tilde{\rho}_{1}^{\diamond},
$$

where $\tilde{\rho}_{0}^{\diamond}$ is a density operator supported on the eigenspace of $\tilde{H}^{\diamond}$ with the maximal eigenvalue, and $\tilde{\rho}_{1}^{\diamond}$ is a density operator supported on the eigenspace of $\tilde{H}^{\diamond}$ with the minimal eigenvalue. The minimization in Eq. (24) ensures that $\tilde{H}^{*}$ of this form can be chosen to obey the constraint Eq. (21). The optimal code would be purifications of $\tilde{\rho}_{0}^{\diamond}$ and $\tilde{\rho}_{1}^{\diamond}$ with orthogonal support in $\mathcal{H}_{A}$. 


\subsection{Ancilla-free quantum error-correcting code}

The above construction of QEC sensing code relies on the noiseless ancilla system. It is yet not clear (i) under what conditions HL scaling can be restored with an ancilla-free QEC code; (ii) whether such code can achieve the same optimal QFI in Eq. (24). These questions can be partially answered in terms of a sufficient condition on the signal Hamiltonian and the Lindblad jump operators ${ }^{41}$.

Theorem 2 (Ancilla-free code under commuting noise) Suppose $H \notin \mathfrak{S}$ and $\left[H, L_{i}\right]=\left[L_{i}, L_{j}\right]=0, \forall i, j$. Then there exists a QEC sensing code without noiseless ancilla that asymptotically recovers the Heisenberg limit in $t$. Moreover, it achieves the same optimal asymptotic QFI [Eq. (24)] offered by noiseless ancillas.

Proof. Without loss of generality, we consider only a 2-dimensional code

$$
\left|0(1)_{\mathrm{L}}\right\rangle=\sum_{k=1}^{d} \sqrt{\beta_{k}^{0(1)}}|k\rangle,
$$

where $\{|k\rangle\}_{k=1}^{d}$ is an orthonormal basis under which $H$ and $L_{i}$ 's are diagonal. Define $d$-dimensional vectors $\mathbf{1}, \boldsymbol{h}, \boldsymbol{\ell}_{i}$, and $\boldsymbol{\ell}_{i j}$ such that $(\mathbf{1})_{k}=1,(\boldsymbol{h})_{k}=\langle k|H| k\rangle,\left(\boldsymbol{\ell}_{\boldsymbol{i}}\right)_{k}=\left\langle k\left|L_{i}\right| k\right\rangle$ and $\left(\boldsymbol{\ell}_{\boldsymbol{i j}}\right)_{k}=\left\langle k\left|L_{i}^{\dagger} L_{j}\right| k\right\rangle$. Define the real subspace $\mathfrak{S}_{\text {diag }}=\operatorname{span}\left\{\mathbf{1}, \operatorname{Re}\left[\boldsymbol{\ell}_{\boldsymbol{i}}\right], \operatorname{Im}\left[\boldsymbol{\ell}_{\boldsymbol{i}}\right], \operatorname{Re}\left[\boldsymbol{\ell}_{\boldsymbol{i} \boldsymbol{j}}\right], \operatorname{Im}\left[\boldsymbol{\ell}_{\boldsymbol{i} \boldsymbol{j}}\right], \forall i, j\right\} \subseteq \mathbb{R}^{d}$. The optimal code can be identified from the optimal solution $\boldsymbol{\beta}^{*}=\boldsymbol{\beta}^{\mathbf{0}}-\boldsymbol{\beta}^{\mathbf{1}}$ of the following $\mathrm{SDP}^{58}$,

$$
\begin{array}{ll}
\operatorname{maximize} & \langle\boldsymbol{\beta}, \boldsymbol{h}\rangle \\
\text { subject to } & \|\boldsymbol{\beta}\|_{1} \leq 2, \text { and }\langle\boldsymbol{\beta}, \boldsymbol{\ell}\rangle=0, \forall \boldsymbol{\ell} \in \mathfrak{S}_{\text {diag }} .
\end{array}
$$

Here $\|\boldsymbol{x}\|_{1}=\sum_{i=1}^{d}\left|x_{i}\right|$ is the one-norm in $\mathbb{R}^{d}$ and $\langle\boldsymbol{x}, \boldsymbol{y}\rangle=\sum_{i=1}^{d} x_{i} y_{i}$ the inner product. Choosing the optimal input quantum state $\left|\psi_{0}\right\rangle=\frac{1}{\sqrt{2}}\left(\left|0_{\mathrm{L}}\right\rangle+\left|1_{\mathrm{L}}\right\rangle\right)$, the QFI is $F\left(\rho_{\omega}(t)\right)=t^{2}\left|\left\langle\boldsymbol{\beta}^{\mathbf{0}}-\boldsymbol{\beta}^{\mathbf{1}}, \boldsymbol{h}\right\rangle\right|^{2}$. Moreover, the optimal value of Eq. (28) is $2 \min _{\ell \in \mathfrak{S}_{\text {diag }}}\|\boldsymbol{h}+\boldsymbol{\ell}\|_{\infty}$ with the argument of the minimum denoted by $\boldsymbol{\ell}^{\diamond}$. Here $\|\cdot\|_{\infty}$ denotes the infinity/max norm, defined as the largest absolute value of elements in a vector. The optimal solution $\boldsymbol{\beta}^{\mathbf{0}(\mathbf{1}) *}$ can be obtained from the constraint that it is in the span of vectors $\boldsymbol{v}$ such that $\left\langle\boldsymbol{v}, \boldsymbol{h}+\boldsymbol{\ell}^{\diamond}\right\rangle$ is the largest (smallest). ${ }^{58}$ In this case,

$$
F\left(\rho_{\omega}(t)\right)=4 t^{2}\left\|\boldsymbol{h}-\mathfrak{S}_{\text {diag }}\right\|_{\infty}^{2}
$$

is the same as $F_{\text {opt }}$ in Eq. (24) for noiseless ancilla. Therefore, we can conclude that $\boldsymbol{\beta}^{\mathbf{0}(\mathbf{1}) *}$ gives the optimal code.

\section{EXAMPLES}

Now we present two explicitly constructed ancilla-free quantum error correcting codes, where the code is defined only in the probe system $\mathcal{H}_{P}{ }^{41}$.

\subsection{Qubits under correlated dephasing noise}

A common sensing scenario involves a quantum sensor composed of $N$ probing qubits with energy gaps proportional to $\omega^{3}$. For such a sensor to be effective, the qubits' energy gaps must depend strongly on $\omega$, which in turn makes them vulnerable to rapid dephasing due to fluctuations in their energies from a noisy environment ${ }^{59-64}$. Assuming for simplicity that each qubit has the same dephasing time $T_{2}$, the generic Markovian dynamics for the sensor is

$$
\frac{d \rho_{P}}{d t}=-i\left[\omega H, \rho_{P}\right]+\frac{1}{2 T_{2}} \sum_{j, k=1}^{N} c_{j k}\left(Z_{j} \rho_{P} Z_{k}-\frac{1}{2}\left\{Z_{j} Z_{k}, \rho_{P}\right\}\right) .
$$

Here, $H=\frac{1}{2} \mathfrak{h} \cdot \boldsymbol{Z}$ where $\mathfrak{h} \in \mathbb{R}^{N}$ and $\boldsymbol{Z}=\left(Z_{1}, \ldots, Z_{N}\right)$ is a vector of Pauli-Z matrices on each site. Qubit $j$ has an energy gap $\omega \mathfrak{h}_{j}$. The correlation matrix $C=\left(c_{j k}\right)_{j, k=1}^{N}$ describing the spatial structure of the noise can be quite general, e.g. depending on their coupling to a nearby fluctuator or a common resonator. In particular, $c_{j k} \in[-1,1]$ describes the correlation between the fluctuations on qubits $j$ and $k$, with $c_{j k}=1,-1$ and 0 signifying full positive, full negative, and the absence of correlations, respectively. 
Eq. (31) can be converted to the form of Eq. (1) by diagonalizing $C\left(C \boldsymbol{v}_{j}=\lambda_{j} \boldsymbol{v}_{j}\right)$ with an orthonormal eigenbasis. Concretely, $L_{j}=\sqrt{\lambda_{j}} \boldsymbol{v}_{j} \cdot \boldsymbol{Z}$ can be viewed as normal modes of the phase noise. The HNLS condition then translates to $\mathfrak{h} \notin \operatorname{col}(C)$, the column space of $C$, which occurs when one normal mode $u$ overlapping with $H$ (i.e., $\boldsymbol{v}_{u} \cdot \mathfrak{h} \neq 0$ ) has a vanishing amplitude, $\lambda_{u}=0$. This occurs generically in the limit of strong spatial noise correlations, provided the noise is not uniformly global ${ }^{39}$. Observe that $\left[H, L_{j}\right]=\left[L_{j}, L_{k}\right]=0$ here, so Theorem 2 guarantees a QEC code without noiseless ancillas saturating the optimal bound in Eq. (24). One such code, for $N \geq 2$, is given by

$$
\left|0_{\mathrm{L}}\right\rangle=\bigotimes_{j=1}^{N}\left(\cos \theta_{j}\left|0_{j}\right\rangle+i \sin \theta_{j}\left|1_{j}\right\rangle\right), \quad\left|1_{\mathrm{L}}\right\rangle=X^{\otimes N}\left|0_{\mathrm{L}}\right\rangle,
$$

where $\boldsymbol{\theta}=\frac{1}{2} \arccos \mathfrak{b}^{\diamond}$, defined element-wise, and $\mathfrak{b}^{\diamond}$ is the solution of the following SDP:

$$
\operatorname{maximize}\langle\mathfrak{b}, \mathfrak{h}\rangle \text {, subject to }\|\mathfrak{b}\|_{\infty} \leq 1, \mathfrak{b} \perp \operatorname{col}(C) \text {. }
$$

For $N=1$, HNLS is not satisfiable for non-zero noise. It is straightforward to show that the code in Eq. (32), with this choice of $\mathfrak{b}^{\diamond}$, satisfies properties Eqs. (10)-(11) and Eq. (13). It works by correcting all non-vanishing noise modes, but leaving a vanishing mode with the maximum overlap with $H$ uncorrected, through which $H$ affects the logical state. Moreover, it achieves the optimal asymptotic QFI [Eq. (24)], in this case:

$$
F_{\text {opt }}(t)=t^{2}\|\mathfrak{h}-\operatorname{col}(C)\|_{1}^{2} .
$$

Remarkably, while the domain of the SDP in Eqs. (28)-(29) has dimension $O\left(2^{N}\right)$, that of Eq. (33) only has dimension $O(N)$ : our ansatz in Eq. (32) renders the QEC code optimization efficient. Note that when

$$
\tilde{\mathfrak{b}}^{\diamond}=\gamma \operatorname{proj}_{\operatorname{ker}(C)} \mathfrak{h},
$$

where $\gamma=\left\|\operatorname{proj} j_{\operatorname{ker}(C)} \mathfrak{h}\right\|_{\infty}^{-1}$. The code using $\boldsymbol{\theta}=\frac{1}{2} \arccos \tilde{\mathfrak{b}}^{\diamond}$ always satisfies properties Eqs. (10)-(11) and Eq. (13), although it needs not saturate the optimal QFI in Eq. (34). In the important case of a single vanishing noise mode [i.e., $\operatorname{rank}(C)=N-1$ ], however, $\tilde{\mathfrak{b}}^{\diamond}$ achieves the optimal QFI.

\subsection{Lossy bosonic channel}

Boson loss is often the dominant decoherence mechanism in a bosonic mode ${ }^{65}$, described by the master equation

$$
\frac{d \rho_{P}}{d t}=-i\left[\sum_{i=1}^{s} \zeta_{i}\left(a^{\dagger} a\right)^{i}, \rho_{P}\right]+\kappa\left(a \rho_{P} a^{\dagger}-\frac{1}{2}\left\{a^{\dagger} a, \rho_{P}\right\}\right)
$$

where $a$ is the annihilation operator and $\kappa$ the boson loss rate. We only consider Hamiltonians that are a function of the boson number $a^{\dagger} a$, applying a cutoff at the $s$-th power, where $s>1$ is a positive integer. We also truncate the boson number at $M$, to keep the system dimension finite. According to the HNLS condition, while $\zeta_{1}$ cannot be sensed at the Heisenberg limit, $\omega:=\zeta_{s}$ asymptotically can.

To sense $\omega$, it is important to filter out all lower-order signals $\sum_{i=1}^{s-1} \zeta_{i}\left(a^{\dagger} a\right)^{i}$ using the QEC code. Therefore, we should use the following modified Lindblad span:

$$
\mathfrak{S}=\operatorname{span}\left\{I, a, a^{\dagger},\left(a^{\dagger} a\right)^{i}, 1 \leq i \leq s-1\right\} .
$$

Note that the boson loss noise is not commuting because $\left[a,\left(a^{\dagger} a\right)^{i}\right] \neq 0$. Still, this type of off-diagonal noise can be tackled by simply ensuring the distance of the supports (non-vanishing terms) of $\left|0_{\mathrm{L}}\right\rangle$ and $\left|1_{\mathrm{L}}\right\rangle$ is at least 3.

To obtain the optimal code, we could solve the SDP in Eqs. (28)-(29). However, when $M$ is sufficiently large, we obtain a near-optimal solution analytically by observing that for large $M$, minimizing $\left\|\left(a^{\dagger} a\right)^{s}-\sum_{i=0}^{s-1} \chi_{i}\left(a^{\dagger} a\right)^{i}\right\|$ over all $\left\{\chi_{i}\right\}_{i=0}^{s-1}$ is equivalent to approximating a $s$-th degree polynomial using an $(s-1)$-degree polynomial. 
The optimal polynomial is the Chebyshev polynomial ${ }^{66}$ and the near-optimal code, that we call the $s$-th order Chebyshev code, is supported by its $\max / \min$ points:

$$
\left|0_{\mathrm{L}}\right\rangle=\sum_{k \text { even }}^{[0, s]} \tilde{c}_{k}\left|\left\lfloor M \sin ^{2}\left(\frac{k \pi}{2 s}\right)\right\rfloor\right\rangle, \quad\left|1_{\mathrm{L}}\right\rangle=\sum_{k \text { odd }}^{[0, s]} \tilde{c}_{k}\left|\left\lfloor M \sin ^{2}\left(\frac{k \pi}{2 s}\right)\right\rfloor\right\rangle,
$$

where $\lfloor x\rfloor$ denotes the largest integer $\leq x$, and $\left|\tilde{c}_{k}\right|^{2}$ can be obtained from solving a linear system of equations of size $O\left(s^{2}\right)$. $\left|\tilde{c}_{k}\right|^{2}$ is approximately equal to $\frac{2}{s}-\frac{1}{s} \delta_{k s}-\frac{1}{s} \delta_{k 0}$ for sufficiently large $M$. It is also interesting to note that the supports of $\left|0_{\mathrm{L}}\right\rangle$ and $\left|1_{\mathrm{L}}\right\rangle$ bears a resemblance to the optimal time intervals in Uhrig dynamical decoupling ${ }^{67}$.

The $s$-th order Chebyshev code corrects the Lindblad span (Eq. (37)) and provides a near optimal asymptotic QFI for $\omega$

$$
F\left(\rho_{\omega}(t)\right) \approx F_{\mathrm{opt}}\left(\rho_{\omega}(t)\right) \approx 16 t^{2}\left(\frac{M}{4}\right)^{2 s}
$$

for sufficiently large $M$.

\section{BEYOND THE HNLS CONDITION}

Generic Markovian noise is full rank, which means that the span $\mathfrak{S}$ is the full Hilbert space $\mathcal{H}_{P}$ of the probe; hence the HNLS criterion of Theorem 1 is violated for any probe Hamiltonian $H$, and asymptotic SQL scaling cannot be surpassed. Therefore, for any Markovian noise model that meets the HNLS criterion, the HL scaling achieved by our QEC code is not robust against generic small perturbations of the noise model.

We should therefore emphasize that a substantial improvement in precision can still be achieved using a QEC code even in cases where HNLS is violated ${ }^{38}$. Consider in particular a Markovian master equation with Lindblad operators divided into two sets $\left\{L_{k}\right\}$ ( $L$-type noise) and $\left\{J_{m}\right\}$ ( $J$-type noise)

$$
\frac{d \rho_{P}}{d t}=-i\left[\omega H, \rho_{P}\right]+\sum_{k}\left(L_{k} \rho_{P} L_{k}^{\dagger}-\frac{1}{2}\left\{L_{k}^{\dagger} L_{k}, \rho_{P}\right\}\right)+\sum_{m}\left(J_{m} \rho_{P} J_{m}^{\dagger}-\frac{1}{2}\left\{L_{m}^{\dagger} L_{m}, \rho_{P}\right\}\right)
$$

where the $J$-type noise is parametrically weak, with noise strength defined by

$$
\epsilon_{J}:=\left\|\sum_{m} J_{m}^{\dagger} J_{m}\right\|
$$

If we use the optimal code that protects against $L$-type noise, then the joint logical state of probe and ancilla evolves according to a modified master equation, with Hamiltonian $H_{\text {eff }}=\Pi_{C} H \Pi_{C}$, and effective Lindblad operators $J_{m, j}$ acting within the code space (the subscript $j$ denotes different error syndromes), where

$$
\left\|\sum_{m, j} J_{m, j}^{\dagger} J_{m, j}\right\| \leq \epsilon_{J}
$$

The state of the error-corrected probe only deviates by a distance $O\left(\epsilon_{J} t\right)$ (in the $L^{1}$ norm) from the (effectively noiseless) evolution in the absence of $J$-type noise. Therefore, using this code, the QFI of the error-corrected probe increases quadratically in time (and the precision $\delta \hat{\omega}$ scales like $1 / t$ ) up until an evolution time $t \propto 1 / \epsilon_{J}$, before crossing over to asymptotic SQL scaling.

When $\epsilon_{J}$ is not infinitesimally small, it is still possible that QEC could enhance estimation precision ${ }^{41}$. To show how, we generalize the dephasing qubits example Sec. 4.1 to this more realistic setting. When HNLS is satisfied, the code in Eq. (32) corrects noise modes with non-zero amplitude $\lambda_{j}>0$, but leaves a mode with $\lambda_{u}=0$ uncorrected. In experiments, the noise correlation matrix $C$ is generically full-rank, meaning that the HNLS condition is not satisfied. Yet, non-trivial noise correlations will generally cause $C$ to have a non-uniform spectrum, yielding some subdominant eigenvalues and corresponding $L_{j}$ 's. It is thus possible to design a code that still accumulates signal at the cost of leaving uncorrected just one subdominant noise mode $\left(\lambda_{u} \approx 0\right)$ through 
an appropriate choice of $\boldsymbol{\theta}$ in Eq. (32). To reach a closed-form expression for the resulting sensitivity, we use $\tilde{\mathfrak{b}}^{\diamond}$ [Eq. (35)] as a starting point rather than an SDP formulation, setting

$$
\boldsymbol{\theta}=\frac{1}{2} \arccos \left(\gamma \boldsymbol{v}_{u}\right)
$$

defined element-wise, where $\gamma=\left\|\boldsymbol{v}_{u}\right\|_{\infty}^{-1}$.

The natural figure of merit for a sensor with uncorrected noise is not the Fisher information: decoherence eventually causes $F\left(\rho_{\omega}(t)\right)$ to decrease, rather than grow unbounded as in Eq. (24). Instead, it is sensitivity, defined as the smallest resolvable signal per unit time ${ }^{3}$. For a single qubit with an energy gap $A \omega$ and dephasing time $T_{2} / B$, the best achievable sensitivity is ${ }^{39,41}$

$$
\eta=\min _{t>0} \frac{1}{\sqrt{F\left(\rho_{\omega}(t)\right) / t}}=\frac{\sqrt{B}}{A} \sqrt{\frac{2 e}{T_{2}}} .
$$

Taking $\mathfrak{h}_{j}=1$ in Eq. (31), each physical qubit $(A=B=1)$ gives $\eta_{1}=\sqrt{2 e / T_{2}} . \quad N$ such qubits operated in parallel give $\eta_{\text {par }}=\eta_{1} / \sqrt{N}$, while for entangled states one could reach $A=N$, often at the cost of an increased $B$. For example, a Greenberger-Horne-Zeilinger (GHZ) sensing scheme with the same $N$ qubits gives

$$
\eta_{\mathrm{GHZ}}=\frac{\left\|D_{C}^{1 / 2} V^{\top} \mathfrak{h}\right\|_{2}}{N} \sqrt{\frac{2 e}{T_{2}}}
$$

where $V=\left(\boldsymbol{v}_{1}, \ldots, \boldsymbol{v}_{N}\right)$ and $D_{C}=\operatorname{diag}\left(\lambda_{1}, \ldots, \lambda_{N}\right) .{ }^{68}$ Note that for uncorrelated noise we have $\left\|D_{C}^{1 / 2} V^{\top} \mathfrak{h}\right\|_{2}=$ $\sqrt{N}$, thus negating any gains from entanglement.

To find the sensitivity offered by the QEC code described above, we compute the sensor's effective Liouvillian, $\mathcal{L}_{\text {eff }}=\mathcal{R} \circ \mathcal{L} \circ \mathcal{P}$, under frequent recoveries $\mathcal{R}$, where $\mathcal{P}(\rho)=P \rho P^{39}$. The usual QEC recovery (i.e., the transpose channel) results in population leakage out of the codespace due to the uncorrected error $L_{u}$ which complicates the analysis ${ }^{27,69}$. To prevent such leakage at the first order, we modify the usual recovery so that the state is returned to the codespace after an error $L_{u}$, though perhaps with a logical error. This modification results in a Markovian, trace-preserving effective dynamics over the two-dimensional codespace, given by $\mathcal{L}_{\text {eff. }}$ Specifically, the sensor's effective dynamics becomes that of a dephasing qubit with $A=\gamma\left|\boldsymbol{v}_{u} \cdot \mathfrak{h}\right|$ and $B=\gamma^{2} \lambda_{u}$, giving $\eta_{\mathrm{QEC}}^{(u)}=\eta_{1} \sqrt{\lambda_{u}} /\left|\boldsymbol{v}_{u} \cdot \mathfrak{h}\right|$. The optimal choice of $u$ is the one that minimizes this quantity, giving:

$$
\eta_{\mathrm{QEC}}=\frac{1}{\left\|D_{C}^{-1 / 2} V^{\top} \mathfrak{h}\right\|_{\infty}} \sqrt{\frac{2 e}{T_{2}}}
$$

valid for arbitrary noise correlation profile $C^{*}$. Eq. (46) identifies the $C$ 's for which this QEC scheme provides enhanced sensitivity over parallel and GHZ sensing. Notice that while HNLS is satisfied only in a measurezero set of $C$ 's, QEC can enhance sensitivity over a much larger set, regardless of whether it can approach the Heisenberg limit in $t$. It admits a broad range of $\eta_{\text {QEC }}$ vs. $N$ scalings due to the critical dependence of $\eta_{\text {QEC }}$ on $C=C(N)$. The same is true of the Fisher information in the HNLS limit as we show in.

\section{DISCUSSION}

Noise limits the precision of quantum sensing. Quantum error correction can suppress the damaging effects of noise, thereby improving the fidelity of quantum information processing and quantum communication, but whether QEC improves the efficacy of quantum sensing depends on the structure of the noise and the signal Hamiltonian. Unless suitable conditions are met, the QEC code that tames the noise might obscure the signal as well, nullifying the advantages of QEC.

${ }^{*} D_{C}^{-1 / 2}$ is undefined when $C$ is singular. In this case, Eq. (46) should be regularized by replacing $D_{C} \rightarrow D_{C}+\epsilon I$, evaluating the norm, then taking $\epsilon \rightarrow 0$. 
Our study of quantum sensing using a noisy probe has focused on whether the precision $\delta$ of parameter estimation scales asymptotically with the total sensing time $t$ as $\delta \propto 1 / t$ (Heisenberg limit) or $\delta \propto 1 / \sqrt{t}$ (standard quantum limit). We have investigated this question in an idealized setting, where the experimentalist has access to noiseless (or correctable) ancillas and can apply quantum controls which are arbitrarily fast and accurate, and we have also assumed that the noise acting on the probe is Markovian. Under these assumptions, we have found the general criterion for HL scaling to be achievable, the Hamiltonian-not-in-Lindblad span (HNLS) criterion. If HNLS is satisfied, a QEC code can be constructed which achieves HL scaling, and if HNLS is violated, then SQL scaling cannot be surpassed. Nonetheless, when HNLS is approximately satisfied, the coherence time of the probe could still be substantially extended under QEC.

The problem of finding the optimal QEC code with the best estimation precision can be formulated as a semidefinite program, which is numerically tractable ${ }^{70}$. The optimal QFI has a pleasing geometrical interpretation, determined by the distance between the Hamiltonian and the Lindblad span under operator norm. Furthermore, using the SDP fomalism we show that when the Hamiltonian and the noise operators all commute, there exist an optimal ancilla-free QEC code, providing a sufficient condition to remove the noiseless ancilla requirement in a realistic setting. We also present two explicit examples of ancilla-free QEC codes: qubits undergoing dephasing and a lossy bosonic mode, which is of practical interest.

Many questions merit further investigation. We have focused on the dichotomy of HL vs. SQL scaling, but it is also worthwhile to characterize constant factor improvements in precision that can be achieved using QEC in cases where HNLS is violated ${ }^{71}$. We should also clarify the role of noiseless ancilla in error-corrected quantum sensing. Commuting noise is sufficient, but not necessary for ancilla-free $\operatorname{codes}^{32,33,49}$ and it is an interesting open problem to refine Theorem 2 into a necessary and sufficient condition. Finally, it is interesting to consider probes subject to non-Markovian noise, in which case, tools such as dynamical decoupling ${ }^{59,67,72,73}$ can mitigate noise. Just as for QEC, we need to balance desirable suppression of the noise against undesirable suppression of the signal in order to formulate the most effective sensing strategy.

\section{ACKNOWLEDGMENTS}

We thank Fernando Brandão, Yanbei Chen, Steve Girvin, Linshu Li, Mikhail Lukin, Changling Zou, Victor Albert, Kyungjoo Noh and Florentin Reiter for inspiring discussions. We acknowledge support from the ARL-CDQI (W911NF-15-2-0067, W911NF-18-2-0237), ARO (W911NF-18-1-0020, W911NF-18-1-0212, W911NF-14-1-0011, W911NF-14-1-0563), ARO MURI (W911NF-16-1-0349, W911NF-15-1-0548), AFOSR MURI (FA9550-14-1-0052, FA9550-15-1-0015), DOE (DE-SC0019406), NSF (EFMA-1640959, EFRIACQUIRE 1641064, EECS1702716), Alfred P. Sloan Foundation (BR2013-049) and the Packard Foundation (2013-39273). The Institute for Quantum Information and Matter is an NSF Physics Frontiers Center with support from the Gordon and Betty Moore Foundation.

\section{REFERENCES}

[1] Giovannetti, V., Lloyd, S., and Maccone, L., "Quantum metrology," Physical review letters 96(1), 010401 (2006).

[2] Giovannetti, V., Lloyd, S., and Maccone, L., "Advances in quantum metrology," Nature photonics 5(4), $222-229$ (2011).

[3] Degen, C. L., Reinhard, F., and Cappellaro, P., "Quantum sensing," Reviews of modern physics 89(3), 035002 (2017).

[4] Caves, C. M., "Quantum-mechanical noise in an interferometer," Physical Review D 23(8), 1693 (1981).

[5] Wineland, D., Bollinger, J., Itano, W., Moore, F., and Heinzen, D., "Spin squeezing and reduced quantum noise in spectroscopy," Physical Review A 46(11), R6797 (1992).

[6] Holland, M. and Burnett, K., "Interferometric detection of optical phase shifts at the heisenberg limit," Physical review letters 71(9), 1355 (1993).

[7] McKenzie, K., Shaddock, D. A., McClelland, D. E., Buchler, B. C., and Lam, P. K., "Experimental demonstration of a squeezing-enhanced power-recycled michelson interferometer for gravitational wave detection," Physical review letters 88(23), 231102 (2002). 
[8] Lee, H., Kok, P., and Dowling, J. P., "A quantum rosetta stone for interferometry," Journal of Modern Optics 49(14-15), 2325-2338 (2002).

[9] Bollinger, J., Itano, W. M., Wineland, D., and Heinzen, D., "Optimal frequency measurements with maximally correlated states," Physical Review A 54(6), R4649 (1996).

[10] Leibfried, D., Barrett, M., Schaetz, T., Britton, J., Chiaverini, J., Itano, W., Jost, J., Langer, C., and Wineland, D., "Toward heisenberg-limited spectroscopy with multiparticle entangled states," Science 304(5676), 1476-1478 (2004).

[11] Valencia, A., Scarcelli, G., and Shih, Y., "Distant clock synchronization using entangled photon pairs," Applied Physics Letters 85(13), 2655-2657 (2004).

[12] Giovannetti, V., Lloyd, S., and Maccone, L., "Quantum-enhanced measurements: beating the standard quantum limit," Science 306(5700), 1330-1336 (2004).

[13] de Burgh, M. and Bartlett, S. D., "Quantum methods for clock synchronization: Beating the standard quantum limit without entanglement," Physical Review A 72(4), 042301 (2005).

[14] Huelga, S. F., Macchiavello, C., Pellizzari, T., Ekert, A. K., Plenio, M. B., and Cirac, J. I., "Improvement of frequency standards with quantum entanglement," Phys. Rev. Lett. 79, 3865-3868 (1997).

[15] Demkowicz-Dobrzański, R., Dorner, U., Smith, B., Lundeen, J., Wasilewski, W., Banaszek, K., and Walmsley, I., "Quantum phase estimation with lossy interferometers," Physical Review A 80(1), 013825 (2009).

[16] Fujiwara, A. and Imai, H., "A fibre bundle over manifolds of quantum channels and its application to quantum statistics," Journal of Physics A: Mathematical and Theoretical 41(25), 255304 (2008).

[17] Escher, B., de Matos Filho, R., and Davidovich, L., "General framework for estimating the ultimate precision limit in noisy quantum-enhanced metrology," Nature Physics 7(5), 406-411 (2011).

[18] Demkowicz-Dobrzański, R., Kołodyński, J., and Guţă, M., "The elusive heisenberg limit in quantumenhanced metrology," Nature Communications 3, 1063 (2012).

[19] Kołodyński, J. and Demkowicz-Dobrzański, R., "Efficient tools for quantum metrology with uncorrelated noise," New Journal of Physics 15(7), 073043 (2013).

[20] Chaves, R., Brask, J., Markiewicz, M., Kołodyński, J., and Acín, A., "Noisy metrology beyond the standard quantum limit," Physical review letters 111(12), 120401 (2013).

[21] Plenio, M. B. and Huelga, S. F., "Sensing in the presence of an observed environment," Physical Review A 93(3), 032123 (2016).

[22] Albarelli, F., Rossi, M. A., Paris, M. G., and Genoni, M. G., "Ultimate limits for quantum magnetometry via time-continuous measurements," New Journal of Physics 19(12), 123011 (2017).

[23] Albarelli, F., Rossi, M. A., Tamascelli, D., and Genoni, M. G., "Restoring heisenberg scaling in noisy quantum metrology by monitoring the environment," arXiv:1803.05891 (2018).

[24] Chin, A. W., Huelga, S. F., and Plenio, M. B., "Quantum metrology in non-markovian environments," Physical review letters 109(23), 233601 (2012).

[25] Smirne, A., Kołodyński, J., Huelga, S. F., and Demkowicz-Dobrzański, R., "Ultimate precision limits for noisy frequency estimation," Physical review letters 116(12), 120801 (2016).

[26] Matsuzaki, Y., Benjamin, S. C., and Fitzsimons, J., "Magnetic field sensing beyond the standard quantum limit under the effect of decoherence," Physical Review A 84(1), 012103 (2011).

[27] Nielsen, M. A. and Chuang, I. L., [Quantum computation and quantum information], Cambridge university press (2010).

[28] Knill, E. and Laflamme, R., "Theory of quantum error-correcting codes," Physical Review A 55(2), 900 (1997).

[29] Gottesman, D., "An introduction to quantum error correction and fault-tolerant quantum computation," in [Quantum information science and its contributions to mathematics, Proceedings of Symposia in Applied Mathematics], 68, 13-58 (2009).

[30] Kessler, E. M., Lovchinsky, I., Sushkov, A. O., and Lukin, M. D., "Quantum error correction for metrology," Physical review letters 112(15), 150802 (2014).

[31] Arrad, G., Vinkler, Y., Aharonov, D., and Retzker, A., "Increasing sensing resolution with error correction," Physical review letters 112(15), 150801 (2014). 
[32] Dür, W., Skotiniotis, M., Froewis, F., and Kraus, B., "Improved quantum metrology using quantum error correction," Physical Review Letters 112(8), 080801 (2014).

[33] Ozeri, R., "Heisenberg limited metrology using quantum error-correction codes.," arXiv:1310.3432 (2013).

[34] Lu, X.-M., Yu, S., and Oh, C., "Robust quantum metrological schemes based on protection of quantum fisher information," Nature communications 6, 7282 (2015).

[35] Matsuzaki, Y. and Benjamin, S., "Magnetic-field sensing with quantum error detection under the effect of energy relaxation," Physical Review A 95(3), 032303 (2017).

[36] Sekatski, P., Skotiniotis, M., Kołodyński, J., and Dür, W., "Quantum metrology with full and fast quantum control," Quantum 1, 27 (2017).

[37] Demkowicz-Dobrzański, R., Czajkowski, J., and Sekatski, P., "Adaptive quantum metrology under general markovian noise," Phys. Rev. X 7, 041009 (2017).

[38] Zhou, S., Zhang, M., Preskill, J., and Jiang, L., "Achieving the heisenberg limit in quantum metrology using quantum error correction," Nature communications 9(1), 78 (2018).

[39] Layden, D. and Cappellaro, P., "Spatial noise filtering through error correction for quantum sensing," npj Quantum Information 4(1), 30 (2018).

[40] Kapourniotis, T. and Datta, A., "Fault-tolerant quantum metrology," arXiv:1807.04267 (2018).

[41] Layden, D., Zhou, S., Cappellaro, P., and Jiang, L., "Ancilla-free quantum error correction codes for quantum metrology," arXiv:1811.01450 (2018).

[42] Gorini, V., Kossakowski, A., and Sudarshan, E. C. G., "Completely positive dynamical semigroups of n-level systems," Journal of Mathematical Physics 17(5), 821-825 (1976).

[43] Lindblad, G., "On the generators of quantum dynamical semigroups," Communications in Mathematical Physics 48(2), 119-130 (1976).

[44] Unden, T., Balasubramanian, P., Louzon, D., Vinkler, Y., Plenio, M. B., Markham, M., Twitchen, D., Stacey, A., Lovchinsky, I., Sushkov, A. O., et al., "Quantum metrology enhanced by repetitive quantum error correction," Physical Review Letters 116(23), 230502 (2016).

[45] Ofek, N., Petrenko, A., Heeres, R., Reinhold, P., Leghtas, Z., Vlastakis, B., Liu, Y., Frunzio, L., Girvin, S., Jiang, L., et al., "Extending the lifetime of a quantum bit with error correction in superconducting circuits," Nature 536(7617), 441-445 (2016).

[46] Heeres, R. W., Reinhold, P., Ofek, N., Frunzio, L., Jiang, L., Devoret, M. H., and Schoelkopf, R. J., "Implementing a universal gate set on a logical qubit encoded in an oscillator," Nature Communications 8(1), 94 (2017).

[47] Rosenblum, S., Gao, Y., Reinhold, P., Wang, C., Axline, C., Frunzio, L., Girvin, S., Jiang, L., Mirrahimi, M., Devoret, M., et al., "A cnot gate between multiphoton qubits encoded in two cavities," Nature communications 9(1), 652 (2018).

[48] Lidar, D. A., Chuang, I. L., and Whaley, K. B., "Decoherence-free subspaces for quantum computation," Physical Review Letters 81(12), 2594 (1998).

[49] Reiter, F., Sørensen, A. S., Zoller, P., and Muschik, C., "Dissipative quantum error correction and application to quantum sensing with trapped ions," Nat. Commun. 8(1), 1822 (2017).

[50] Lihm, J.-M., Noh, K., and Fischer, U. R., "Implementation-independent sufficient condition of the knilllaflamme type for the autonomous protection of logical qudits by strong engineered dissipation," Physical Review A 98(1), 012317 (2018).

[51] Demkowicz-Dobrzański, R. and Maccone, L., "Using entanglement against noise in quantum metrology," Physical review letters 113(25), 250801 (2014).

[52] Yuan, H., "Sequential feedback scheme outperforms the parallel scheme for hamiltonian parameter estimation," Phys. Rev. Lett. 117, 160801 (2016).

[53] Helstrom, C. W., [Quantum detection and estimation theory], Academic press (1976).

[54] Braunstein, S. L. and Caves, C. M., "Statistical distance and the geometry of quantum states," Physical Review Letters 72(22), 3439 (1994).

[55] Holevo, A. S., [Probabilistic and statistical aspects of quantum theory], vol. 1, Springer Science \& Business Media (2011).

[56] Kolodynski, J., Precision bounds in noisy quantum metrology, PhD thesis, University of Warsaw (2014). 
[57] Bény, C., "Perturbative quantum error correction," Physical review letters 107(8), 080501 (2011).

[58] Boyd, S. and Vandenberghe, L., [Convex optimization], Cambridge university press (2004).

[59] Biercuk, M. J., Uys, H., VanDevender, A. P., Shiga, N., Itano, W. M., and Bollinger, J. J., "Optimized dynamical decoupling in a model quantum memory," Nature 458(7241), 996-1000 (2009).

[60] Witzel, W. M., Carroll, M. S., Morello, A., Cywiński, L., and Das Sarma, S., "Electron spin decoherence in isotope-enriched silicon," Phys. Rev. Lett. 105, 187602 (2010).

[61] Bluhm, H., Foletti, S., Neder, I., Rudner, M., Mahalu, D., Umansky, V., and Yacoby, A., "Dephasing time of gaas electron-spin qubits coupled to a nuclear bath exceeding $200 \mu$ s," Nature Physics 7(2), 109-113 (2011).

[62] Doherty, M. W., Manson, N. B., Delaney, P., Jelezko, F., Wrachtrup, J., and Hollenberg, L. C., "The nitrogen-vacancy colour centre in diamond," Physics Reports 528(1), 1 - 45 (2013). The nitrogen-vacancy colour centre in diamond.

[63] Muhonen, J. T., Dehollain, J. P., Laucht, A., Hudson, F. E., Kalra, R., Sekiguchi, T., Itoh, K. M., Jamieson, D. N., McCallum, J. C., Dzurak, A. S., et al., "Storing quantum information for 30 seconds in a nanoelectronic device," Nature nanotechnology 9(12), 986-991 (2014).

[64] Orgiazzi, J.-L., Deng, C., Layden, D., Marchildon, R., Kitapli, F., Shen, F., Bal, M., Ong, F. R., and Lupascu, A., "Flux qubits in a planar circuit quantum electrodynamics architecture: Quantum control and decoherence," Phys. Rev. B 93, 104518 (2016).

[65] Chuang, I. L., Leung, D. W., and Yamamoto, Y., "Bosonic quantum codes for amplitude damping," Physical Review A 56(2), 1114 (1997).

[66] Mason, J. C. and Handscomb, D. C., [Chebyshev polynomials], Chapman and Hall/CRC (2002).

[67] Uhrig, G. S., "Keeping a quantum bit alive by optimized $\pi$-pulse sequences," Physical Review Letters 98(10), 100504 (2007).

[68] Greenberger, D. M., Horne, M. A., and Zeilinger, A., [Going Beyond Bell's Theorem], 69-72, Springer Netherlands, Dordrecht (1989).

[69] Lidar, D. and Brun, T., [Quantum Error Correction], Cambridge University Press (2013).

[70] Grant, M. and Boyd, S., "Cvx: Matlab software for disciplined convex programming."

[71] Herrera-Martí, D. A., Gefen, T., Aharonov, D., Katz, N., and Retzker, A., "Quantum error-correctionenhanced magnetometer overcoming the limit imposed by relaxation," Physical review letters 115(20), 200501 (2015).

[72] Viola, L., Knill, E., and Lloyd, S., "Dynamical decoupling of open quantum systems," Physical Review Letters 82(12), 2417 (1999).

[73] Maze, J., Stanwix, P., Hodges, J., Hong, S., Taylor, J., Cappellaro, P., Jiang, L., Dutt, M. G., Togan, E., Zibrov, A., et al., "Nanoscale magnetic sensing with an individual electronic spin in diamond," $\mathrm{Na}$ ture 455(7213), 644-647 (2008). 